\title{
Depósitos de Calda de Pulverização nas faces Adaxial e ABAXIAL DE FOlHAS DE Eichhornia crassipes DisPOSTAS EM DIFERENTES ÂNGULOS ${ }^{1}$
}

\author{
Spray Solution Deposits on Adaxial and Abaxial Faces of Eichhornia crassipes Leaves \\ Arranged Under Different Angles
}

MARCHI, S.R. ${ }^{2}$, MARTINS, D. ${ }^{3}$, COSTA, N.V. ${ }^{2}$, CARBONARI, C.A. ${ }^{2}$ e TERRA, M.A. ${ }^{2}$

\begin{abstract}
RESUMO - Estudos foram conduzidos com o objetivo de avaliar os depósitos de gotas pulverizadas através de dois tipos de pontas sobre as faces adaxial e abaxial de folhas de Eichhornia crassipes dispostas em diferentes ângulos. No primeiro estudo, os tratamentos foram dispostos no esquema fatorial 2x4x7: dois tipos de pontas (TX12 e XR11002VS), quatro ângulos verticais $\left(0^{\circ}, 30^{\circ}, 60^{\circ}\right.$ e $\left.90^{\circ}\right)$ e sete ângulos horizontais $\left(0^{\circ}, 15^{\circ}, 30^{\circ}, 45^{\circ}, 60^{\circ}\right.$, $75^{\circ} \mathrm{e} 90^{\circ}$ ). No ângulo vertical de $90^{\circ}$ a lâmina foliar foi posicionada paralelamente ao sentido de deslocamento do jato de pulverização; e no ângulo horizontal de $90^{\circ}$ a folha foi posicionada perpendicularmente ao plano do solo. Como traçadores, foram utilizadas soluções de 1.000 ppm do corante Azul FDC-1 e 3.500 ppm do corante Amarelo Tartrasina para as pontas tipo leque e cone, respectivamente. No segundo estudo, os tratamentos foram dispostos no esquema fatorial 2x2x3: dois tipos de pontas (TX12 e XR11002VS), dois tipos de calda (com e sem Aterbane BR a $0,5 \%)$ e três ângulos horizontais $\left(0^{\circ}, 45^{\circ}\right.$ e $\left.90^{\circ}\right)$. Adotou-se o ângulo vertical de $90^{\circ}$ como padrão para todos os tratamentos. Soluções de $1.000 \mathrm{ppm}$ do corante Azul FDC-1 e 3.500 ppm do corante Amarelo Tartrasina foram utilizadas como traçadores para a calda com e sem surfatante, respectivamente. Os resultados do primeiro estudo revelaram que os depósitos observados em toda a folha e na face adaxial das lâminas foliares de aguapé diminuíram à medida que se aumentou a angulação vertical, independentemente da ponta utilizada. Os menores depósitos sempre estiveram relacionados ao ângulo vertical de $90^{\circ}$, independentemente do ângulo horizontal utilizado. Não foi detectado nenhum depósito na face abaxial das folhas de aguapé em todas as combinações dos fatores estudados. No segundo estudo também foram observados os mesmos decréscimos no volume de calda depositado em toda a folha e na face adaxial à medida que se aumentou a angulação vertical das lâminas foliares. As duas pontas proporcionaram depósitos nulos na face abaxial quando o surfatante não foi utilizado; entretanto, a adição do surfatante à calda incrementou significativamente os depósitos nesta face da folha do aguapé.
\end{abstract}

Palavras-chave: deposição de gotas, aguapé, arquitetura foliar, surfatante, tecnologia de aplicação.

\begin{abstract}
This study aimed to evaluate the deposition of drops using two nozzle types over Eich hornia crassipes leaves. The first trial was arranged in a factorial design $2 \times 4 \times 7$, as follows: 2 nozzle types (TX12 and XR11002VS), 4 vertical angles $\left(0^{\circ}, 30^{\circ}, 60^{\circ}\right.$ and $\left.90^{\circ}\right)$ and 7 horizontal angles $\left(0^{\circ}, 15^{\circ}, 30^{\circ}, 45^{\circ}, 60^{\circ}, 75^{\circ}\right.$ and $\left.90^{\circ}\right)$. The laminar leaf was set up parallel to the jet spraying movement direction at a vertical angle of $90^{\circ}$, and perpendicular to the soil plane at a horizontal angle of $90^{\circ}$. Solutions of dyes FD\&C Blue no. 1 at 1,000 ppm and FD\&C Yellow no. 5 at 3.500 ppm were used as spraying tracers for flat fan and cone nozzles, respectively. The second trial was set up as a factorial design 2x2x3, being 2 nozzle types (TX12 and XR11002VS), 2 spray solution conditions (with and without Aterbane BR at $0.5 \% \mathrm{v} / \mathrm{v})$ and 4 horizontal angles $\left(0^{\circ}, 45^{\circ}\right.$ and $\left.90^{\circ}\right)$.
\end{abstract}

1 Recebido para publicação em 7.1.2005 e na forma revisada em 10.3.2005.

2 Eng.-Agr., discente de Pós-graduação, Dep. Produção Vegetal-Agricultura, UNESP/FCA <marchi@fca.unesp.br>; ${ }^{3}$ Prof. Livre Docente., Dep. Produção Vegetal-Agricultura, UNESP/FCA, Caixa Postal 237, 18603-970 Botucatu-SP. 
The vertical angle of $90^{\circ}$ was assigned as pattern to all the treatments. Solutions of dyes FD\&C Blue no. 1 at 1,000 ppm and FD\&C Yellow no. 5 at 3.500 ppm were used as spraying tracers with or without surfactant, respectively. The results obtained from the first trial showed that the spray solution deposits observed on the whole leaf and on the foliar adaxial face of the laminar leaves of water hyacinth decreased as the vertical angle became higher, regardless of the nozzle type used. The lowest spray solution deposits were always related to the vertical angle of $90^{\circ}$, regardless of the horizontal angle adopted. No spray solution deposit was detected on the abaxial face of water hyacinth leaf at the combinations of the factors studied. In the second trial the same spray solution deposit was observed to decrease the whole leaf and adaxial faces as the vertical angle increased. Both nozzle types provided null and void deposits on the abaxial face when the surfactant was not used. However, the surfactant addition to the spray solution provided significant increases on the abaxial face of water hyacinth leaves.

Key words: drop deposition, water hyacinth, foliar architecture, surfactant, spraying technology.

\section{INTRODUÇÃO}

Os aspectos positivos e negativos da presença de plantas aquáticas nos diversos ambientes aquáticos, as alterações ambientais que levam à proliferação de forma desorganizada de populações e os prejuízos causados por estas plantas aos diversos usos dos corpos hídricos têm sido amplamente abordados por diversos pesquisadores brasileiros (Esteves, 1998; Pitelli, 1998; Martins et al., 1999; Tanaka, 1998; Velini, 2000; Thomas, 2002; Tanaka et al., 2002; Alves et al., 2003; Martins et al., 2003).

Em grande parte dos estudos envolvendo plantas daninhas de ambiente aquático, o aguapé (Eichhornia crassipes) tem recebido especial atenção, por se tratar de uma espécie de ocorrência cosmopolita e que apresenta grande capacidade de produção de biomassa verde em ambientes eutrofizados (Holm et al., 1991). Particularmente, as folhas do aguapé estão dispostas em roseta, sendo normalmente elipticas ou reniformes. Os pecíolos são esponjosos e, dependendo da circunstância, inflados, o que auxilia na flutuação da planta. Tal estrutura também é denominada de pulvino (Kissmann \& Groth, 1997).

Tanto o pecíolo quanto o pulvino apresentam a peculiaridade de alterar suas posições durante o ciclo de desenvolvimento vegetativo do aguapé, modificando totalmente a arquitetura da planta. Observando-se infestações de aguapé em diferentes locais, é possivel verificar que regiões periféricas das populações apresentam plantas flutuantes mais jovens e, por conseguinte, pecíolos e pulvinos dispostos em posições próximas à horizontal. Já as plantas localizadas no centro das populações apresentam o pulvino estiolado (não-inflado) e, juntamente com o limbo foliar, disposto em uma posição mais próxima à vertical.

Essa última forma de arquitetura é típica de plantas mais velhas, de populações mais densas e que se encontram estacionadas ou ancoradas em um mesmo local durante longos periodos. Assumindo uma arquitetura vertical, as folhas tendem a posicionar seus limbos em direção única devido à ação de ventos e, por conseguinte, conseguem se dispersar a partir do ponto onde estavam estacionadas ou ancoradas (Holm et al., 1991).

Dessa forma, as populações de aguapé podem se deslocar através da ação do vento e, fazendo-se analogia, o conjunto de limbo e pecíolo pode atuar como velas propulsoras. De modo geral, a maioria das populações de aguapé presentes nos corpos hídricas apresenta arquiteturas próximas à vertical e, dependendo do ângulo de observação, pode conduzir a possiveis erros de interpretação quanto ao indice de cobertura vegetal da área onde se localizam.

Além da dispersão de plantas, a arquitetura de folhas pode ainda influenciar a quantidade dos depósitos de calda pulverizada sobre as plantas de aguapé, uma vez que a posição do limbo foliar em relação à direção do jato de pulverização pode favorecer ou mesmo prejudicar o contato entre a gota e a superficie da folha. Teoricamente, folhas orientadas na 
posição horizontal são mais eficientes na captura de gotas que aquelas orientadas na posição vertical (Spillman, 1984; Wirth et al., 1991; Gerard et al., 1998; Richardson \& Newton, 2000). Essas diferenças nos depósitos de calda em função da arquitetura de folhas do aguapé também poderiam explicar os diversos resultados obtidos no controle químico desta espécie encontrados na literatura, principalmente naqueles estudos em que não se utilizaram surfatantes na calda de pulverização (Martins et al., 2002; Neves et al., 2002).

Assim, o objetivo deste trabalho foi estudar os depósitos de gotas pulverizadas por meio de dois tipos de pontas, com e sem adjuvante, sobre as faces adaxial e abaxial das folhas de E. crassipes dispostas em diferentes ângulos horizontais e verticais.

\section{MATERIAL E MÉTODOS}

Dois estudos foram conduzidos no Núcleo de Pesquisas Avançadas em Matologia (NUPAM), pertencente ao Departamento de Produção Vegetal/Agricultura da Faculdade de Ciências Agronômicas/UNESP, campus de Botucatu/SP.

No primeiro estudo, os tratamentos foram dispostos no esquema fatorial $2 \times 4 \times 7$, sendo duas pontas de pulverização (TX12 e XR11002VS), quatro ângulos verticais $\left(0^{\circ}, 30^{\circ}\right.$, $60^{\circ}$ e $\left.90^{\circ}\right)$ e sete ângulos horizontais $\left(0^{\circ}, 15^{\circ}\right.$, $30^{\circ}, 45^{\circ}, 60^{\circ}, 75^{\circ}$ e $90^{\circ}$ ). No ângulo vertical de $90^{\circ}$, a lâmina foliar foi posicionada paralelamente ao sentido de deslocamento do jato de pulverização e, no ângulo horizontal de $90^{\circ}$, ela foi posicionada perpendicularmente ao solo. Folhas recém-cortadas das plantas de aguapé foram fixadas individualmente em um conjunto composto por presilha de metal acoplada a uma haste de arame flexivel. As diversas posições na vertical foram obtidas girando-se a presilha de metal em torno da haste flexivel, e as diversas posições na horizontal foram obtidas pela dobra na haste de arame flexível.

A aplicação da calda utilizando-se a ponta do tipo leque foi realizada na mesma folha de aguapé 45 minutos antes da aplicação da calda com a ponta do tipo cone. Esses procedimentos tiveram os objetivos de utilizar o mesmo alvo para estudar os depósitos de calda proporcionados pelos dois tipos de ponta de pulverização, além de permitir a total secagem da primeira calda pulverizada sobre as folhas e, conseqüentemente, evitar possiveis perdas por escorrimento na segunda aplicação.

Foi utilizado um pulverizador estacionário a pressão constante de ar comprimido, regulado para um consumo de calda de $200 \mathrm{~L} \mathrm{ha}^{-1}$. Soluções de 1.000 ppm do corante Azul FDC-1 e $3.500 \mathrm{ppm}$ do corante Amarelo Tartrasina foram utilizadas como traçadores para as pontas tipo leque e cone, respectivamente. As concentrações dos corantes adotadas neste estudo estão de acordo com aquelas sugeridas por Souza (2002), em que ambos os corantes podem ser utilizados como traçadores no estudo quantitativo de situações distintas de pulverizações efetuadas em alvo único.

O depósito total dos corantes nas folhas de aguapé foi avaliado através da extração destes com $20 \mathrm{~mL}$ de água destilada imediatamente após o término da segunda aplicação. A extração dos corantes nas faces abaxial e adaxial das folhas de aguapé foi feita através de equipamento desenvolvido no NUPAM, o qual é constituído de uma alça metálica com formato de pinça, munida em suas extremidades de tubos plásticos contendo $15 \mathrm{~mL}$ de água destilada. Foram feitas duas extrações em cada uma das superficies foliares, uma vez que a abertura do tubo plástico possibilita a lavagem de uma área circular equivalente a $2,379 \mathrm{~cm}^{2}$. Cabe salientar que as avaliações dos depósitos totais nas folhas foram realizadas em lâminas foliares distintas daquelas em que foram efetuadas as avaliações das partes adaxial e abaxial das folhas de aguapé. As novas soluções obtidas foram acondicionadas em frascos plásticos e destinadas para posterior leitura em espectrofotômetro.

No segundo estudo, os tratamentos foram dispostos no esquema fatorial $2 \times 2 \times 3$, sendo duas pontas de pulverização (TX12 e XR11002VS), dois tipos de calda (com e sem Aterbane $\mathrm{BR}$ a $0,5 \%)$ e somente três ângulos horizontais $\left(0^{\circ}, 45^{\circ}\right.$ e $\left.90^{\circ}\right)$. Adotou-se o ângulo vertical de $90^{\circ}$ como padrão para todos os tratamentos. Utilizaram-se folhas planas de aguapé que não apresentassem concavidades nas suas bordas. Lamínulas de vidro foram dispostas no ângulo de $90^{\circ}$, tanto na horizontal como na vertical, e tiveram suas faces

Planta Daninha, Viçosa-MG, v. 23, n. 2, p. 321-328, 2005 
aleatoriamente estipuladas como adaxial e abaxial para servirem como testemunha.

A aplicação da calda, com surfatante, foi realizada na mesma folha de aguapé 45 minutos após ter sido feita a aplicação da calda sem surfatante, pelos motivos já descritos no primeiro estudo. As aplicações foram feitas utilizando-se soluções de $1.000 \mathrm{ppm}$ do corante Azul Brilhante FDC-1 e 3.500 ppm do corante Amarelo Tartrasina como traçadores para a calda com e sem surfatante, respectivamente. As pulverizações e a extração dos corantes depositados no alvo e nas faces adaxial e abaxial, tanto das folhas de aguapé como nas laminulas, foram efetuadas através dos mesmos procedimentos adotados no primeiro estudo.

Todas as soluções obtidas nos dois estudos foram prontamente analisadas em espectrofotômetro UV-visível de duplo feixe, modelo Cintra 20, operando com 3,0 $\mathrm{mm}$ de caminho óptico, cujos resultados em absorbância nos comprimentos de onda de 630 e $427 \mathrm{~nm}$ para os corantes Azul Brilhante e Amarelo Tartrasina, respectivamente, foram transformados em ppm através da confecção de curvaspadrão a partir de concentrações conhecidas. Especialmente para o corante Azul Brilhante, tomou-se o cuidado de verificar sua absorbância também no comprimento de onda de $427 \mathrm{~nm}$, com o objetivo de evitar a sobreposição de leituras para este corante (Souza, 2002).

As áreas de cada limbo foliar de aguapé utilizado nos dois estudos, estimada pela equação proposta por Marchi \& Pitelli (2003), e da lamínula foram utilizadas nos cálculos dos

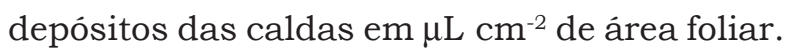
Os resultados, em $\mu \mathrm{L} \mathrm{cm}^{-2}$, obtidos nos estudos foram submetidos à análise de variância pelo teste $\mathrm{F}$ e as médias dos tratamentos comparadas pelo teste t a $5 \%$ de probabilidade.

\section{RESULTADOS E DISCUSSÃO}

Na Tabela 1 estão apresentados os depósitos totais (folha inteira) e na face adaxial das folhas de aguapé proporcionados pelas pontas TX12 e XR11002VS. Pode-se observar que os depósitos em toda a folha e na face adaxial diferiram significativamente à medida que se alterou a angulação das folhas, independentemente da ponta utilizada. Os maiores depósitos sempre estiveram relacionados com os ângulos de $0^{\circ}$ tanto na horizontal quanto na vertical e tenderam a diminuir à medida que o ângulo se aproximou de $90^{\circ}$.

De forma semelhante, a interação significativa entre os ângulos também revela que os totais de depósito de calda encontrados na folha e na face adaxial diminuíram gradativamente à proporção que se aumentou a combinação entre os ângulos horizontais e verticais das folhas, independentemente do tipo de ponta utilizado.

Os resultados obtidos no primeiro estudo estão de acordo com a afirmação de que o ângulo da folha pode ter um efeito significante sobre a deposição da calda pulverizada na superficie foliar. Segundo Spillman (1984), plantas planófilas, ou seja, espécies que possuem folhas aproximadamente paralelas à superfície do solo e com pequeno ângulo foliar, retêm maior quantidade de gotas pulverizadas. Já as plantas erectófilas, as quais possuem grandes ângulos foliares, como as gramíneas, às vezes apresentam menor interceptação e retenção da calda pulverizada. Richardson \& Newton (2000) verificaram que a deposição da calda pulverizada sobre Pteridium aquilinum foi comparativamente superior àquela observada sobre Arctostaphylos patula. Os autores comentam que as diferenças são devidas à arquitetura das plantas, cujas folhas formam ângulos aproximadamente de $0^{\circ}$ e $72^{\circ}$ com a superficie do solo, respectivamente.

Gerard et al. (1998), analisando a relação entre a arquitetura da planta de cevada e a interceptação da calda de pulverização, também verificaram que a concentração da calda na folha aumenta da folha-bandeira para as folhas mais velhas. Os autores concluem que a folha-bandeira possui maior angulação e que tal ângulo tende a se aproximar da horizontal à medida que a folha vai envelhecendo, o que favorece a deposição de gotas. Comparativamente, esse processo ocorre de maneira inversa nas plantas de aguapé, visto que estas tendem a adquirir uma arquitetura próxima da vertical à proporção que as folhas envelhecem. Esse fenômeno pode influenciar decisivamente o depósito da calda pulverizada sobre as diversas populações de aguapé. 
Tabela 1 - Depósitos totais e na face adaxial proporcionados pelas pontas TX12 e XR10002VS em folhas de E. crassipes. Botucatu-SP, 2003

\begin{tabular}{|c|c|c|c|c|c|c|c|c|}
\hline \multicolumn{9}{|c|}{ Ponta TX12 } \\
\hline \multicolumn{5}{|c|}{ Depósitos na folha ${ }^{1 /}\left(\mu \mathrm{L} \mathrm{cm}^{-2}\right.$ de área foliar $)$} & \multicolumn{4}{|c|}{ Depósitos na face adaxial ${ }^{2 /}\left(\mu \mathrm{L} \mathrm{cm}^{-2}\right.$ de área foliar $)$} \\
\hline \multirow{2}{*}{$\begin{array}{c}\text { Ângulo } \\
\text { horizontal }\end{array}$} & \multicolumn{4}{|c|}{ Ângulo vertical } & \multicolumn{4}{|c|}{ Ângulo vertical } \\
\hline & $0^{\circ}$ & $30^{\circ}$ & $60^{\circ}$ & $90^{\circ}$ & $0^{\circ}$ & $30^{\circ}$ & $60^{\circ}$ & $90^{\circ}$ \\
\hline $0^{\circ}$ & $1,304 \mathrm{Aa}$ & $1,186 \mathrm{Aa}$ & $1,001 \mathrm{Bab}$ & $0,882 \mathrm{Bb}$ & $1,851 \mathrm{Aa}$ & $1,663 \mathrm{Aab}$ & $1,411 \mathrm{Bab}$ & $1,026 \mathrm{Ca}$ \\
\hline $15^{\circ}$ & $1,315 \mathrm{Aa}$ & $1,199 \mathrm{Aa}$ & $0,977 \mathrm{Bab}$ & $0,786 \mathrm{Cab}$ & $1,601 \mathrm{Abc}$ & $1,590 \mathrm{ABabc}$ & $1,385 \mathrm{BCab}$ & $1,200 \mathrm{Ca}$ \\
\hline $30^{\circ}$ & $1,259 \mathrm{Aa}$ & $1,142 \mathrm{Aa}$ & $0,939 \mathrm{Bab}$ & $0,732 \mathrm{Cb}$ & $1,663 \mathrm{Aab}$ & $1,736 \mathrm{ABa}$ & $1,478 \mathrm{Ba}$ & $1,103 \mathrm{Ca}$ \\
\hline $45^{\circ}$ & $1,116 \mathrm{Ab}$ & $1,139 \mathrm{Aa}$ & $1,034 \mathrm{Aa}$ & $0,788 \mathrm{Bab}$ & $1,535 \mathrm{Abc}$ & $1,449 \mathrm{ABcd}$ & $1,249 \mathrm{BCbc}$ & $1,167 \mathrm{Ca}$ \\
\hline $60^{\circ}$ & $1,109 \mathrm{Ab}$ & $1,073 \mathrm{Aa}$ & $0,937 \mathrm{Bab}$ & $0,739 \mathrm{Cb}$ & $1,428 \mathrm{Acd}$ & $1,458 \mathrm{Abc}$ & $1,264 \mathrm{Abc}$ & $1,021 \mathrm{Ba}$ \\
\hline $75^{\circ}$ & $0,910 \mathrm{Ac}$ & $0,936 \mathrm{Ab}$ & $0,942 \mathrm{Aab}$ & $0,757 \mathrm{Bab}$ & 1,322 Ade & $1,245 \mathrm{ABde}$ & $1,341 \mathrm{Aabc}$ & $1,078 \mathrm{Ba}$ \\
\hline $90^{\circ}$ & $0,797 \mathrm{Ac}$ & $0,848 \mathrm{Ab}$ & $0,902 \mathrm{Ab}$ & $0,840 \mathrm{Aab}$ & $1,178 \mathrm{Ae}$ & $1,157 \mathrm{Ae}$ & $1,148 \mathrm{Ac}$ & $1,053 \mathrm{Aa}$ \\
\hline \multicolumn{9}{|c|}{ Ponta XR11002VS } \\
\hline $0^{\circ}$ & $1,330 \mathrm{Aab}$ & $1,135 \mathrm{Bbc}$ & $0,948 \mathrm{Ca}$ & $0,990 \mathrm{Ca}$ & $1,865 \mathrm{Aa}$ & $1,580 \mathrm{Ba}$ & $1,252 \mathrm{Ca}$ & $1,001 \mathrm{Da}$ \\
\hline $15^{\circ}$ & $1,367 \mathrm{Aab}$ & $1,229 \mathrm{Bab}$ & $1,036 \mathrm{Ca}$ & $0,884 \mathrm{Dabc}$ & $1,552 \mathrm{Ab}$ & $1,527 \mathrm{ABab}$ & $1,330 \mathrm{Ba}$ & $1,116 \mathrm{Ca}$ \\
\hline $30^{\circ}$ & $1,390 \mathrm{Aa}$ & $1,297 \mathrm{Aa}$ & $0,977 \mathrm{Ba}$ & $0,759 \mathrm{Cc}$ & $1,613 \mathrm{Ab}$ & $1,654 \mathrm{Aa}$ & $1,334 \mathrm{Ba}$ & $1,033 \mathrm{Ca}$ \\
\hline $45^{\circ}$ & $1,196 \mathrm{Ac}$ & $1,130 \mathrm{~b} \mathrm{Ac}$ & $1,075 \mathrm{Aa}$ & $0,838 \mathrm{Bbc}$ & $1,449 \mathrm{Abc}$ & $1,456 \mathrm{Aab}$ & 1,192 Bab & $1,110 \mathrm{Ba}$ \\
\hline $60^{\circ}$ & $1,242 \mathrm{Ab}$ & $1,082 \mathrm{Bcd}$ & $0,994 \mathrm{Cb}$ & $0,836 \mathrm{Cbc}$ & $1,344 \mathrm{Acd}$ & $1,356 \mathrm{Abc}$ & $1,168 \mathrm{ABab}$ & $1,000 \mathrm{Ba}$ \\
\hline $75^{\circ}$ & $1,005 \mathrm{Ad}$ & 0,986 Ade & $0,996 \mathrm{Aa}$ & $0,823 \mathrm{Bc}$ & 1,138 Ade & 1,149Acd & 1,216Aab & $1,016 \mathrm{Aa}$ \\
\hline $90^{\circ}$ & $0,844 \mathrm{Be}$ & $0,863 \mathrm{ABe}$ & $0,979 \mathrm{Ba}$ & $0,964 \mathrm{Abab}$ & $1,048 \mathrm{Ae}$ & $1,042 \mathrm{Ad}$ & $1,021 \mathrm{Ab}$ & $1,001 \mathrm{Aa}$ \\
\hline F horizontal $(\mathrm{H})$ & \multicolumn{4}{|c|}{$27,02^{* *}$} & \multicolumn{4}{|c|}{$29,625^{* *}$} \\
\hline F vertical $(\mathrm{V})$ & \multicolumn{4}{|c|}{$129,81 * *$} & \multicolumn{4}{|c|}{$86,157 * *$} \\
\hline F ponta $(\mathrm{P})$ & \multicolumn{4}{|c|}{$21,10^{* *}$} & \multicolumn{4}{|c|}{$16,245^{* *}$} \\
\hline $\mathrm{FH} \times \mathrm{V}$ & \multicolumn{4}{|c|}{$10,73 * *$} & \multicolumn{4}{|c|}{$5,466^{* *}$} \\
\hline $\mathrm{F} \mathrm{H} \times \mathrm{P}$ & \multicolumn{4}{|c|}{$0,62^{\mathrm{NS}}$} & \multicolumn{4}{|c|}{$0,223^{\mathrm{NS}}$} \\
\hline $\mathrm{FV} \times \mathrm{P}$ & \multicolumn{4}{|c|}{$1,22^{\mathrm{NS}}$} & \multicolumn{4}{|c|}{$0,333^{\mathrm{NS}}$} \\
\hline $\mathrm{FH} \times \mathrm{V} \times \mathrm{P}$ & \multicolumn{4}{|c|}{$0,47^{\mathrm{NS}}$} & \multicolumn{4}{|c|}{$0,162^{\mathrm{NS}}$} \\
\hline CV $(\%)$ & \multicolumn{4}{|c|}{9,2} & \multicolumn{4}{|c|}{11,3} \\
\hline d.m.s. & \multicolumn{4}{|c|}{0,1304} & \multicolumn{4}{|c|}{0,2074} \\
\hline
\end{tabular}

** significativo a $1 \%$ de probabilidade; * significativo a $5 \%$ de probabilidade; ${ }^{\text {NS }}$ não-significativo; ${ }^{1 /}$ Média transformada em raiz de $\mathrm{x} ;{ }^{2 /}$ Médias transformadas em raiz de $\mathrm{x}+1$.

Médias seguidas de mesma letra minúscula na coluna ou de mesma letra maiúscula na linha não diferem estatisticamente entre si pelo teste $\mathrm{t}$ $(\mathrm{p}>0,05)$.

Ainda, na Tabela 1 , pode-se notar que os depósitos de calda totais observados na folha, dentro de cada combinação de ângulos, foram quantitativamente inferiores àqueles observados na face adaxial. Esse fato provavelmente esteja associado à distribuição irregular de gotas ao longo de toda a superficie superior da folha, que pode ter interferido na extração dos corantes nos diferentes pontos amostrados. Os efeitos da distribuição irregular de gotas possivelmente foram diluídos na avaliação dos depósitos totais na folha, uma vez que a extração foi feita através da lavagem total do limbo foliar. Além disso, os depósitos de calda pulverizada na face abaxial das folhas de aguapé foram praticamente nulos nas diversas combinações de ângulo horizontal e vertical estudadas e, dessa forma, não contribuíram na quantidade total de calda depositada em todo o limbo foliar.

A deposição ou retenção praticamente nula de gotas na face abaxial da folha de aguapé observada neste estudo provavelmente esteja associada à presença de substâncias de natureza hidrofóbica (Kern et al., 1997; Kirkwood, 1999) ou, então, a pequenas concavidades nas bordas dos limbos foliares, os quais estariam influenciando negativamente o contato entre a gota e a superficie foliar.

Os depósitos de calda totais, sem e com surfatante, na folha e na face adaxial de folhas de aguapé observados no segundo estudo estão 
apresentados na Tabela 2. A adição do surfatante à calda aumentou significativamente a quantidade dos depósitos de calda totais na folha e na face adaxial, independentemente do ângulo e do tipo de ponta utilizada. Entretanto, o uso de surfatante não influenciou a deposição de calda nas lamínulas, uma vez que as quantidades depositadas em toda a laminula quanto na sua respectiva face adaxial foram estatisticamente semelhantes.

Os efeitos da adição do surfatante à calda pulverizada foram marcantes nos depósitos observados na face abaxial das folhas do aguapé (Tabela 3). As pontas TX 12 e XR11002VS novamente proporcionaram depósitos praticamente nulos nesta superfície foliar na ausência de surfatante. Contudo, a adição do surfatante proporcionou depósitos significativos na face abaxial das folhas. Ainda na Tabela 3, é possivel notar que o uso ou não do surfatante não alterou as quantidades de depósitos observadas na face abaxial das lamínulas previamente estabelecidas. Costa (2004), em estudos sobre as características da anatomia e da superfície foliar de plantas daninhas aquáticas, verificou que a utilização de soluções de diferentes tensões superficiais proporcionadas pela adição de diversos surfatantes aumentou significativamente a superficie de molhamento das faces adaxial e abaxial das folhas de aguapé, quando comparadas com água pura. $\mathrm{O}$ autor relata ainda que somente a solução contendo Aterbane BR a $0,5 \% \mathrm{v} \mathrm{v}^{-1}$ proporcionou diferenças estatísticas entre as faces adaxial e abaxial quanto à superficie de molhamento, sendo estas maiores na página inferior do limbo foliar.

Cardoso et al. (2003), avaliando o controle químico de seis acessos de Eichhornia crassipes coletados em reservatórios do Estado de São Paulo, utilizando o herbicida glyphosate nas doses de 1.680 e 3.360 g e.a. ha ${ }^{-1}+0,5 \%$ v v$^{-1}$ do adjuvante Extravon, observaram notas de controle variando entre 91 e $100 \%$ aos 14 dias após a aplicação, constatando a eficiência do herbicida glyphosate no controle desta espécie. Esses resultados são similares aos encontrados por Martins et al. (1999), os quais, avaliando o efeito do glyphosate (3.360 g e.a. ha ${ }^{-1}$ ) sem adjuvante no controle de Eichhornia crassipes

Tabela 2 - Depósitos de calda de pulverização, sem e com surfatante, observados na folha toda e na face adaxial de E. crassipes. Botucatu-SP, 2003

\begin{tabular}{|c|c|c|c|c|c|c|c|c|}
\hline \multicolumn{9}{|c|}{ Ponta TX12 } \\
\hline \multirow[b]{3}{*}{ Calda $^{2 /}$} & \multicolumn{4}{|c|}{ Depósitos na folha ${ }^{1 /}\left(\mu \mathrm{L} \mathrm{cm}^{-2}\right.$ de área foliar $)$} & \multicolumn{4}{|c|}{ Depósitos na face adaxial ${ }^{1 /}\left(\mu \mathrm{L} \mathrm{cm}^{-2}\right.$ de área foliar $)$} \\
\hline & \multicolumn{4}{|c|}{ Ângulo horizontal } & \multicolumn{4}{|c|}{ Ângulo horizontal } \\
\hline & $0^{\circ}$ & $45^{\circ}$ & $90^{\circ}$ & Lâmina & $0^{\circ}$ & $45^{\circ}$ & $90^{\circ}$ & Lâmina \\
\hline Sem & $0,844 \mathrm{Aa}$ & $0,857 \mathrm{Aa}$ & 0,681 Aab & $0,882 \mathrm{Ab}$ & $0,585 \mathrm{Aa}$ & $0,693 \mathrm{Aa}$ & $1,052 \mathrm{Aa}$ & $1,026 \mathrm{Aa}$ \\
\hline Com & $1,403 \mathrm{Ab}$ & $1,420 \mathrm{Ab}$ & $1,118 \mathrm{Bb}$ & $0,786 \mathrm{Cab}$ & $1,306 \mathrm{Aa}$ & $1,387 \mathrm{Ab}$ & $1,530 \mathrm{Aa}$ & $1,200 \mathrm{Aa}$ \\
\hline \multicolumn{9}{|c|}{ Ponta XR 11002 VS } \\
\hline Sem & $0,945 \mathrm{Aa}$ & $1,006 \mathrm{Aa}$ & $0,971 \mathrm{Aa}$ & $0,990 \mathrm{Aa}$ & $0,861 \mathrm{Aa}$ & $1,325 \mathrm{Aa}$ & $1,307 \mathrm{Aa}$ & $1,000 \mathrm{Aa}$ \\
\hline Com & $1,449 \mathrm{Ab}$ & $1,625 \mathrm{Ab}$ & $1,539 \mathrm{Ab}$ & $0,884 \mathrm{Ba}$ & $1,366 \mathrm{Ba}$ & $2,049 \mathrm{Ab}$ & $1,899 \mathrm{Aa}$ & $1,116 \mathrm{Ba}$ \\
\hline F ângulo (A) & \multicolumn{4}{|c|}{$2,81^{*}$} & \multicolumn{4}{|c|}{$7,99 * *$} \\
\hline $\mathrm{F}$ calda (C) & \multicolumn{4}{|c|}{$110,18 * *$} & \multicolumn{4}{|c|}{$29,37 * *$} \\
\hline F ponta $(\mathrm{P})$ & \multicolumn{4}{|c|}{$15,35 * *$} & \multicolumn{4}{|c|}{$15,77^{* *}$} \\
\hline $\mathrm{FA} \times \mathrm{C}$ & \multicolumn{4}{|c|}{$0,26^{\mathrm{NS}}$} & \multicolumn{4}{|c|}{$0,43^{\mathrm{NS}}$} \\
\hline $\mathrm{FA} \times \mathrm{P}$ & \multicolumn{4}{|c|}{$2,55^{*}$} & \multicolumn{4}{|c|}{$1,14^{*}$} \\
\hline FCXP & \multicolumn{4}{|c|}{$0,19^{\mathrm{NS}}$} & \multicolumn{4}{|c|}{$0,09^{\mathrm{NS}}$} \\
\hline $\mathrm{FAxC \times P}$ & \multicolumn{4}{|c|}{$0,27^{\mathrm{NS}}$} & \multicolumn{4}{|c|}{$0,01^{\mathrm{NS}}$} \\
\hline $\mathrm{CV}(\%)$ & \multicolumn{4}{|c|}{15,5} & \multicolumn{4}{|c|}{29,2} \\
\hline d.m.s. & \multicolumn{4}{|c|}{0,2565} & \multicolumn{4}{|c|}{0,5262} \\
\hline
\end{tabular}

** significativo a $1 \%$ de probabilidade; * significativo a $5 \%$ de probabilidade; ${ }^{\text {NS }}$ não-significativo; ${ }^{1 /}$ Média transformada em raiz de $\mathrm{x} ;{ }^{2 /}$ Aterbane BR a $0,5 \% \mathrm{v} / \mathrm{v}$.

Médias seguidas de mesma letra minúscula na coluna ou de mesma letra maiúscula na linha não diferem estatisticamente entre si pelo teste $\mathrm{t}$ $(\mathrm{p}>0,05)$. 
Tabela 3 - Depósitos de calda de pulverização (em $\mu \mathrm{L} \mathrm{cm}^{-2}$ ), sem e com surfatante, observados na face abaxial das folhas de E. crassipes. Botucatu-SP, 2003

\begin{tabular}{|c|c|c|c|c|c|c|c|c|}
\hline \multicolumn{5}{|c|}{ Ponta TX 12} & \multicolumn{4}{|c|}{ Ponta XR11002VS } \\
\hline \multirow[b]{2}{*}{$\mathrm{Calda}^{2 /}$} & \multicolumn{4}{|c|}{ Ângulo horizontal ${ }^{1 /}$} & \multicolumn{4}{|c|}{ Ângulo horizontal $^{1 /}$} \\
\hline & $0^{\circ}$ & $45^{\circ}$ & $90^{\circ}$ & Lâmina & $0^{\circ}$ & $45^{\circ}$ & $90^{\circ}$ & Lâmina \\
\hline Sem & $\begin{array}{l}1,001 \mathrm{Aa} \\
(0,0020)\end{array}$ & $\begin{array}{l}1,000 \mathrm{Aa} \\
(0,0001)\end{array}$ & $\begin{array}{l}1,000 \mathrm{Aa} \\
(0,0001)\end{array}$ & $\begin{array}{l}1,372 \mathrm{Bb} \\
(0,8823)\end{array}$ & $\begin{array}{c}1,001 \mathrm{Aa} \\
(0,0020)\end{array}$ & $\begin{array}{l}1,001 \mathrm{Aa} \\
(0,0020)\end{array}$ & $\begin{array}{l}1,000 \mathrm{Aa} \\
(0,0001)\end{array}$ & $\begin{array}{l}1,423 \mathrm{Ba} \\
(1,0249)\end{array}$ \\
\hline Com & $\begin{array}{l}1,838 \mathrm{Ab} \\
(2,3782)\end{array}$ & $\begin{array}{c}1,699 \mathrm{Abb} \\
(1,8866)\end{array}$ & $\begin{array}{c}1,530 \mathrm{BCb} \\
(1,3409)\end{array}$ & $\begin{array}{l}1,336 \mathrm{Cb} \\
(0,7849)\end{array}$ & $\begin{array}{l}1,951 \mathrm{Ab} \\
(2,8064)\end{array}$ & $\begin{array}{l}1,602 \mathrm{Bb} \\
(1,5664)\end{array}$ & $\begin{array}{l}1,255 \mathrm{Cb} \\
(0,5750)\end{array}$ & $\begin{array}{c}1,483 \mathrm{BCa} \\
(1,1993)\end{array}$ \\
\hline F ângulo (A) & \multicolumn{8}{|c|}{$5,94 *$} \\
\hline F calda $(\mathrm{C})$ & \multicolumn{8}{|c|}{$117,62 * *$} \\
\hline F ponta (P) & \multicolumn{8}{|c|}{$0,53^{\mathrm{NS}}$} \\
\hline $\mathrm{FAxC}$ & \multicolumn{8}{|c|}{$5,92 * *$} \\
\hline $\mathrm{FA} \times \mathrm{P}$ & \multicolumn{8}{|c|}{$0,89^{\mathrm{NS}}$} \\
\hline $\mathrm{FCxP}$ & \multicolumn{8}{|c|}{$0,53^{\mathrm{NS}}$} \\
\hline $\mathrm{FA} \times \mathrm{C} \times \mathrm{P}$ & \multicolumn{8}{|c|}{$0,89^{\mathrm{NS}}$} \\
\hline CV $(\%)$ & \multicolumn{8}{|c|}{15,6} \\
\hline d.m.s. & \multicolumn{8}{|c|}{0,2957} \\
\hline
\end{tabular}

** significativo a $1 \%$ de probabilidade; * significativo a $5 \%$ de probabilidade; ${ }^{\text {NS }}$ não-significativo; ${ }^{1 /}$ Média transformada em raiz de $\mathrm{x}+1$ (médias originais entre parênteses); ${ }^{2 /}$ Aterbane BR a $0,5 \% \mathrm{v} / \mathrm{v}$. Médias seguidas de mesma letra minúscula na coluna ou de mesma letra maiúscula na linha não diferem estatisticamente entre si pelo teste $t(p>0,05)$.

sob condições controladas, obtiveram médias superiores a $90 \%$ de controle aos 35 dias após a aplicação.

\section{LITERATURA CITADA}

ALVES, E. et al. Avaliações fisiológicas e bioquímicas de plantas de aguapé (Eichhornia crassipes) cultivadas com níveis excessivos de nutrientes. Planta Daninha, v. 21, p. 27-35, 2003. (Edição especial)

CARDOSO, L. R.; MARTINS, D.; TERRA, M. A. Sensibilidade a herbicidas de acessos de aguapé coletados em reservatórios do Estado de São Paulo. Planta Daninha, v. 21, p. 61-67, 2003. (Edição especial).

COSTA, N. V. Caracterização da anatomia e da superfície foliar de plantas daninhas aquáticas e efeito sobre a deposição de gotas de pulverização. 2004. $92 \mathrm{f}$. Dissertação (Mestrado em Agronomia/Agricultura) Universidade Estadual Paulista, Botucatu, 2004.

ESTEVES, F. A. Fundamentos de limnologia. Rio de Janeiro: Interciência, FINEP, 1998. 575 p.

GERARD, A. J. M. et al. Towards predicting pesticide deposition from plant phenology; a study in spring barley. Pestic. Sci., v. 53, p. 252-262, 1998.

HOLM, L.G. et al. The world's worst weeds. Malabar: Krieger Publishing Company, 1991. 609 p.
KERN, A. J.; JAKCSON, L. L; DRYER, W. E. Fat acid and wax biosynthesis in susceptible and triallate-resistance Avena fatua L. Pestic. Sci., v. 51, p. 21-26, 1997.

KIRKWOOD, R. C. Recent developments in our understanding of the plant cuticle as a barrier to foliar uptake of pesticides. Pestic. Sci., v. 55, p. 69-77, 1999.

KISSMANN, K.; GROTH, D. Plantas infestantes e nocivas. 2.ed. São Paulo: BASF Brasileira, 1997. 824 p.

MARCHI, S. R.; PITELLI, R. A. Estimativa da área foliar de plantas daninhas de ambiente aquático. Planta Daninha, v. 21, p. 109-112, 2003. (Edição especial)

MARTINS D. et al. Controle químico de plantas daninhas aquáticas em condições controladas - caixa d'água. Planta Daninha, v. 17, p. 289 -296, 1999.

MARTINS, D. et al. Controle químico de Pistia stratiotes, Eichhornia crassipes e Salvinia molesta em caixas d'água. Planta Daninha, v. 20, p. 83-88, 2002. (Edição especial)

MARTINS, D. et al. Ocorrência de plantas aquáticas nos reservatórios da Light-RJ. Planta Daninha, v. 21, p. 105108, 2003. (Edição especial)

NEVES, T.; FOLONI, L. L.; PITELLI, R. A. Controle químico do aguapé (Eichhornia crassipes). Planta Daninha, v. 20, p. 89-97, 2002. (Edição especial)

Planta Daninha, Viçosa-MG, v. 23, n. 2, p. 321-328, 2005 
PALLADINI, L. A. Metodologia para avaliação da deposição em pulverizações. 2000. $111 \mathrm{f}$. Tese (Doutorado em Proteção de Plantas) - Universidade Estadual Paulista, Botucatu, 2000.

PITELLI, R. A. Macrófitas aquáticas do Brasil. In: WORKSHOP DE CONTROLE DE PLANTAS AQUÁtICAS, 1998, Brasília. Anais... Brasília: 1998. p. 16-17.

RICHARDSON, B.; NEWTON, M. Spray deposition within plant canopies. N. Z. P. Protec., v. 53, p. 248-252, 2000 .

SOUZA, R.T. Efeito da eletrização de gotas sobre a variabilidade dos depósitos de pulverização e eficácia do glyphosate no controle de plantas daninhas da cultura da soja. 2002. 69 f. Tese (Doutorado em Proteção de Plantas) - Universidade Estadual Paulista, Botucatu, 2002.

SPILLMAN, J. J. Spray impaction, retention and adhesion: an introduction to basic characteristics. Pestic. Sci., v. 15, p. 97-106, 1984.
TANAKA, R. H. Prejuízos provocados pelas plantas aquáticas. In: WORKSHOP DE CONTROLE DE PLANTAS AQUÁTICAS, 1998, Brasília. Anais... Brasília: 1998. p. 36-38.

TANAKA, R. H. et al. Ocorrência de plantas daninhas nos reservatórios da Companhia Energética de São Paulo. Planta Daninha, v. 20, p. 101-111, 2002. (Edição especial)

THOMAS, S. M. Fatores ecológicos associados à colonização e ao desenvolvimento de macrófitas aquáticas e desafios de manejo. Planta Daninha, v. 20, p. 21-33, 2002. (Edição especial)

VELINI, E. D. Controle de plantas daninhas aquáticas. In: CONGRESSO BRASILEIRO DA CIÊNCIA DAS

PLANTAS DANINHAS, 22., 2000, Foz do Iguaçu. Palestras... Foz do Iguaçu: Sociedade Brasileira da Ciência das Plantas Daninhas, 2000. p. 137-147.

WIRTH, W.; STORP, S.; JACOBENSEN, W. Mechanisms controlling leaf retention of agricultural spray conditions. Pestic. Sci., v. 31, p. 411-420, 1991. 